\title{
Reconnaissance constitutionnelle des droits et libertés en France à la fin du XVIIIème et XIXème siècles
}

L'histoire constitutionnelle de la France représente un champ d'étude très spécifique, tant par l'extrême richesse que la diversité des instruments constitutionnels expérimentés. Une des caractéristiques essentielles du développement politique de la France dans la période traitrée réside dans la grande variété des mouvements politiques et sociaux qui ont émaillés les XVIIIèmeet XIXèmesiècles. A la fin du XVIIIème siècle, la société française fut divisée entre partisans de l'instauration d'une forme de gouvernement monarchique ou ceux favorables à l'instauration d'un gouvernement républicain. Et, ces luttes politiques distinguèrent la France par le fait que, plus souvent que dans d'autres pays occidentaux comme la Grande-Bretagne et les États-Unis, elles influèrent directement sur la structure de l'État. L'affrontement des différentes classes sociales prédétermina objectivement la dynamique des régimes politiques, puisque l'objet principal de cette lutte portait périodiquement sur la forme elle-même de gouvernement, sa reconnaissance constitutionnelle, le système des institutions étatiques et juridiques, la régulation des droits et libertés. Depuis la fin du XVIIIème siècle, la reconnaissance et la protection des droitshumains et civiquesoccupentdoncune place prépondérantedans de nombreuses constitutions françaises.

Le premier document ayantvaleurconstitutionnelleetgarantissant les droits et libertésfondamentales, fut la Déclaration des droits de l'Homme et du Citoyen (ci-après dénommée «la Déclaration»), adoptée le 26 août 1789. La Déclarationjeta les bases du constitutionnalismefrançaisetinfluença le développementconstitutionnel de nombreux pays. Par son essence ainsiquel'intention de l'Assembléeconstituante à l'origine de sarédaction, la Déclaration constitua alorsaussibien un manifest qu'uncontrat public.

Cette déclaration constitue un petit document, quant à son volume (17 articles au total), qui énonce l'ensemble de droits et libertés fondamentaux les plus importantes. Les auteurs de la Déclaration remplirent alors plusieurs objectifs, qui furent mis en ouvre 
avec succès. Tout d'abord, ce fut la proclamation de vérités simples, évidentes et indiscutables. Deuxièmement, la Déclaration fut la base de la future Constitution. Troisièmement, le texte de la Déclaration fut ouvert à la lecture de tous, et tous les Français purent se familiariser avec les droits qui y sont expressément énoncés. Enfin, les auteurs créèrent un document qui, à bien des égards, fut très en avance sur son temps, puisque les droits, énoncés dans la Déclaration, portent un caractère universel ${ }^{1}$. Cette Déclaration précéda la première Constitution française de 1791.

Malgré une période de temps extrêmement courte quant aux débats portant sur la nouvelle Déclaration (du 20 au 26 aồt 1789), et l'affrontement de différentes conceptions, ses auteurs purent construire un concept d'une cohérence suffisante pour l'affirmation et la reconnaissance des droits et des libertés ${ }^{2}$. Il convient de noter que la Déclaration concerna non seulement les droits de l'Homme mais aussi les droits du Citoyen ; c'est à dire, comme Léon Duguit l'a noté, les droits de l'individu, étant donné qu'y furent proclamés «non seulement les droits appartenant à l'Homme en théorie, mais aussi les droits vraiment reconnus et garantis par la société »3.

Le garant du respect des droits de l'Homme et du Citoyen en vertu de la Constitution de 1791, fut le pouvoir législatif qui «ne peut faire de lois, entravant la mise en ceuvre des droits naturels et civils... ${ }^{4}$.

Le droit constitutionnel moderne de la France note, quant à lui, que la portée juridique de la Déclaration dépend d'au moins deux facteurs. Tout d'abord, de la présence textuelle des droits et des libertés dans la Constitution. Deuxièmement, «de leur nature propre et de la forme de leurénoncé» ${ }^{5}$.

Mais cette Déclaration fut également confirmée par le fait qu'elle fait toujours partie du « Bloc de Constitutionnalité » de la Vème République.

Actuellement, l'unité constitutionnelle est constituée de normes sur les bases desquelles le Conseil constitutionnel contrôle la constitutionnalité des lois et des traités, et se compose de 6 éléments:

- la Constitutionde 1958,

- la Déclaration des droits de l'Homme et du citoyen de 1789,

- le Préambule de la Constitution de 1946,

1 Voir les détails :Morange J. La Déclaration des Droits de l'Homme et du Citoyen. Paris, 1988.

2 Les auteurs de la Déclaration furent influencés, principalement, par la théorie du droit naturel, les points de vues de John Locke, C.-L. Montesquieu, J.-J. Rousseau, les physiocrates français, ainsi que l'expérience de la construction de la nation américaine.

3 Дюги Л. Конституционное право. Общая теория государства. М., 2014. С. 312.

4 Constitution de 1791//http://www.conseil-constitutionnel.fr/conseil-constitutionnel/francais/ la-constitution/les-constitutions-de-la-france/constitution-de-1791.5082.html

5 Chantebout B. Droit constitutionnel. Paris, 2013. 凶. 39-40. 
- Charte de l'environnement de 2004, qui concernent le Préambule de la Constitution de 1958.

- Les principesfondamentauxreconnus par les lois de la République

- lesprincipesquiontvaleurconstitutionnelledéclarésparleConseilconstitutionnel' ${ }^{6}$.

Ilconvient de noterque la question de la validité de la Déclaration de 1789 resta un point discuté pendant une longue période en France. Le facteurdéterminantfut la décision du Conseilconstitutionnel du 27 décembre 1973. Le Conseil constitutionnel considéraque les dispositions de la loi de finances pour 1974 s'opposaient au principed'égalité des citoyensdevant la loi, car elle établissaitune discrimination enverscertainescatégories de personnes. Ainsi, le Conseilconstitutionnel fit référence au texte de la Déclaration pour la première foisdanssa jurisprudence. Ainsi, le Conseil constitutionnel formula l'un des principes fondamentaux reconnus par les lois de la Vème République et reconnut la validité de la Déclaration. Comme M. Morabito le nota, la Déclaration « acquit un caractère sacré et l'importance d'un catéchisme politique, qui ne peut être changée »?.

En 1793, pendant la dictature jacobine, une deuxième Déclaration des droits de l'Homme et du Citoyen (ci-après dénommée «la Déclaration de 1793») futadoptéedans l'histoire de la France. La Déclaration de 1793 proclama le bonheur général en tant que but de la société, et la garantie faite « à l'homme la jouissance de sesdro its naturels et imprescriptibles» en tant qu'objectif de gouvernement. Ces droits comprirent «l'égalité, la liberté, la sécurité et la propriété » ${ }^{8}$. En comparaison avec la Déclaration de 1789 , il y eut un changement : en lieu et place de la "résistance à l'oppression », la liste des droits naturels et imprescriptibles incluait maintenant " l'égalité ».

Une innovation significative fut l'inclusion d'un bloc de droits sociaux dans la Déclaration de 1793. Il s'agissait textuellement de la charité publique envers les pauvres, les chômeurs et les personnes handicapées, de l'éducation nationale, ainsi que d'une disposition stipulant comme suit, que "Nulle contribution ne peutêtre établieque pour l'utilité générale.»"

Pour la première fois dans l'histoire constitutionnelle de la France, le droit de "résistance à l'oppression» fut stipulé avec une conception plus radicale que dans la Déclaration d'indépendance des États-Unis. La Déclaration de 1793 indiqua que « quand le gouvernement porte atteinte aux droits du peuple, le soulèvement est le droit et le devoir sacré d'urgence pour le peuple et pour chacune de ses parties ${ }^{10}$.

6 http://www.legifrance.gouv.fr/Droit-francais/Guide-de-legistique/I.-Conception-destextes/1.3.-Hierarchie-des-normes/1.3.1.-Differentes-normes

7 Morabito M. Histoire constitutionnelle de la France de 1789 à nos jours. Paris, 2012.囚. 57.

8 Déclaration des Droits de l'Hommeet du Citoyen de 1793, article 1er.

9 Déclaration des Droits de l'Hommeet du Citoyen de 1793, article 20.

10 Constitution du 24 juin 1793 // http://www.conseil-constitutionnel.fr/conseil-constitutionnel/ francais/la-constitution/les-constitutions-de-la-france/constitution-du-24-juin-1793.5084. 
Deux ans plus tard, suivant les traditions constitutionnelles, la Convention Thermidorienne élabora et adopta non seulement une Constitution, mais aussi la « Déclaration des droits et devoirs de l'Homme et du Citoyen » du 5 fructidor an III (ci-après dénommée "la Déclaration de l'an III»).

Les droits de l'homme comprirent alors "la liberté, l'égalité, la sûreté et la propriété". Ainsi, le législateur de l'an III répéta la liste énoncée dans la Déclaration de 1793. Cependant, ces droits ne furent pas définis en tant que «naturels et inaliénables ", mais comme "les droits de l'homme dans la société ». À cet égard, il convient de noter l'interprétation faite de l'égalité, qui " consiste dans le fait que la loi est égale pour tous, dans les cas où elle protège, comme dans les cas où elle punit. L'égalité n'autorise aucune distinction fondée sur la naissance, aucun pouvoir héréditaire ». Ainsi, il ne s'agit pas d'une "égalité naturelle ", mais d'une "égalité civile ». Selon F.-A. Boissy d'Anglas, l'un des auteurs de la Constitution de l'an III, : " l'égalité civile - c'est tout ce qu'un homme raisonnable peut prétendre. L'égalité absolue est une chimère ; pour quelle existe, il est nécessaire qu'il y ait une pleine égalité dans les capacités mentales, la vertu, les forces physiques, l'éducation et les chances de toutes les personnes $" 1$.

Le droit de résister à l'oppression et le droit du peuple à la révolte ne furent pas mentionnés. Cela fut conditionné par l'approche textuelle faite par les auteurs de la Déclaration de l'an III : "Nous en (du texte de la Déclaration) avons soigneusement enlevé toutes ces axiomes anarchistes, recueillies par la tyrannie, qui voulaient tout tourner pour asservir tous; ces maximes dégoûtantes, cette violation de tous les principes et le renversement de tous les droits qui permettent à chaque individu criminel d'effectuer des attaques contre la société dans son ensemble, donnent à la minorité agitée et rebelle un privilège à violer les décisions pacifiques et justes de la majorité du peuple, qui seul doit être souverain $»^{12}$. En conséquence, «l'égalité de tous les citoyens » établit la base principale de la souveraineté du Peupledans la Déclaration de l'an III.

Pour la première fois, une section distincte de la Déclaration fut consacrée aux devoirs de l'Homme et du Citoyen, qui y furent formulés en deux phrases : "Ne faites pas à autrui ce que vous ne voulez pas quion vous fasse. Faites constamment aux autres le bien que vous voulez recevoir d'eux $\gg{ }^{13}$.

Après l'arrivée au pouvoir de Napoléon Bonaparte, la Constitution de l'an VIII fut adoptée. Pour la première fois dans l'histoire constitutionnelle de la France, la Constitution de l'an VIII ne fut pas précédée par une Déclaration des droits et libertés. Seul le

html

11 Citation: БовыкинД.Ю. Революцияокончена? Итоги термидора. М., 2005. С. 125.

12 Документы истории Великой французской революции. Т.1. М. 1990. С 299.

13 Constitution du 5 Fructidor An III // http://www.conseil-constitutionnel.fr/conseil-constitutionnel/francais/la-constitution/les-constitutions-de-la-france/constitution-du-5-fructidoran-iii.5086.html. 
chapitre VII, intitulé « Dispositions Générales » mentionne l'inviolabilité du domicile (art. 76), le droit de recours individuel (art. 83), ainsi que des garanties contre les arrestations arbitraires (art. 77-82). Il convient de noter que la Loi fondamentale de l'an VIII, en général, contient très peu des énoncés déclaratifs à caractère fondamental. L'exception fut le premier article, qui déclara : "La République française est unie et indivisible ${ }^{14}$.

Le Sénatus-consulte organique du 16 thermidor an $X$, ainsi que le Sénatus-consulte organique du 28 floréal an XII, nénoncèrent ultérieurement pas de droits et de libertés. Cependant, à partir de 1804, l'empereur agit en tant que garant de leur respect. Conformément à l'art. 53 du Sénatus-consulte organique du 28 floréal an XII, l'empereur jura de « respecter et faire respecter les lois relatives au Concordat et la liberté de religion; respecter et se conformer à l'égalité des droits, des libertés politiques et civiles... "15.La création de deux comités du Sénat, qui furent responsables de « la préservation de la liberté individuelle » et de la liberté de la presse furent également prévue.

Après la restauration de la dynastie des Bourbons en 1814, la Charte octroyée par Louis XVIII fut adoptée. Le premier article de la Charte, consacré au droit public français, confirma les principes d'égalité civile, d'intégrité de la personne, de liberté de la presse, de liberté d'opinion et de religion, qui furent fixés dans les années de la révolution. La Charte proclama l'inviolabilité des droits de propriété : "Tous les types de propriété sont inviolables, n'excluant pas la propriété dite nationale : la loi ne fait aucune distinction entre elles $»^{16}$. Cette disposition constitutionnelle fut extrêmement importante, car elle affecta les intérêts financiers de la majorité écrasante des Français.

Selon A. D. Gradovsky, le législateur russe bien connu : "La Charte octroyée par Louis XVIII, représenta une tentative... de réconciliation des principes révolutionnaires avec les établissements historiques dans le pays. Le constitutionnalisme, qui fut en 1791 le fruit de la révolution, était maintenant sanctifié par la Charte, délivrée par le Roi, libre de ses droits. En outre, elle octroya à la Nation de tels droits et une participation à la gestion, qui pourraient presque être conciliés avec les principes de la monarchie ${ }^{17}$.

Pendant sa deuxième gouvernance des «100 jours », Napoléon approuva le texte de la nouvelle Constitution, connut comme " l'Acte additionnel à la Constitution de l'Empire » (ci-après dénommé "l'Acte additionnel »). Napoléon insista personnellement pour que le

14 Constitution du 22 Frimaire An VIII // http://www.conseil-constitutionnel.fr/conseil-constitutionnel/francais/la-constitution/les-constitutions-de-la-france/constitution-du-22-frimaire-an-viii.5087.html

15 Constitution de l'An XII - Empire - 28 floréal An XII // http://www.conseil-constitutionnel. $\mathrm{fr} /$ conseil-constitutionnel/francais/la-constitution/les-constitutions-de-la-france/constitution-de-1-an-xii-empire-28-floreal-an-xii.5090.html

16 Charte constitutionnelle du 4 juin 1814 // http://www.conseil-constitutionnel.fr/conseil-constitutionnel/francais/la-constitution/les-constitutions-de-la-france/charte-constitutionnelle-du4-juin-1814.5102.html.

17 Градовский А.Д. Собрание сочинений. Т. 4. СПб., 1900. С. 547. 
texte de l'Acte additionnel contienne des références à la Constitution de l'an VIII et au Sénatus-consulte organique du 16 thermidor an X, ainsi qu'au Sénatus-consulte organique du 28 floréal de l'an XII. Ainsi, toutes les normes constitutionnelles, depuis 1799, qui ne furent pas modifiées par l'Acte additionnel, furent de nouveau en vigueur. Cela concerna pleinement les droits et libertés de l'homme et du citoyen.

Toutefois, les articles spéciaux (section 6 de l'Acte additionnel) garantirent l'égalité de tous les Français devant la loi, l'intégrité personnelle, la liberté de religion, l'inviolabilité de la propriété, la liberté de la presse et le droit de pétition. Ainsi, l'Acte additionnel accorda plus d'attention aux droits de l'homme que tous les actes constitutionnels antérieurs adoptés sous Napoléon. Les articles sur les droits humains de la Charte de 1830, qui fut une version révisée de la Charte 1814, ne furent pas changés.

Après la proclamation de la Seconde République, la Constitution de 1848 fut adoptée. La Loi fondamentale de 1848 ne dit rien à propos des droits naturels et contient un grand nombre des dispositions déclaratives. Malgré le fait qu'un chapitre particulier dans la Constitution fut consacré aux droits des citoyens, leur respect ne fut pas garanti, en substance : "L'Assemblée nationale confie la protection de la Constitution et des droits, accordés par elle, au dévouement et au patriotisme de tous les Français $»^{18}$ (art. 110 de la Constitution de 1848).

Le 10 décembre 1848, les élections présidentielles de la Deuxième République eurent lieu. Louis Napoléon Bonaparte (le neveu de Napoléon I) remporta les élections. En 1852, une nouvelle Constitution fut adoptée. Le premier article contint la formule en blanc suivante : "Les principes fondamentaux, déclarés en 1789, servant en tant que la base du droit public français, sont reconnus et confirmés ». Devant un manque évident de clarté, cet article soulève une question : fait-il référence aux principes révolutionnaires ou à ceux énoncés dans la Déclaration de de 1789 ? Les droits et libertés ne furent pas attribués textuellement, toutefois, en vertu de l'article 26, le Sénat eut le droit de s'opposer à la promulgation des lois, qui contredisent ou affectent la religion, la morale, la liberté religieuse, la liberté individuelle, l'égalité devant la loi, l'inviolabilité de la propriété et le principe de l'inamovibilité des juges.

Le 21 mai 1870, au crépuscule du Second Empire, le Sénatus-consulte organique, fixant la Constitution de l'Empire, contenant le libellé similaire à la Constitution de 1852, fut adoptée : "Les principes fondamentaux, déclarés en 1789, servant en tant que base du droit public français, sont reconnus et confirmés. » (art.1) ${ }^{19}$. Cependant, ce Sénatus-consulte orga-

18 Constitution de 1848, IIe République // http://www.conseil-constitutionnel.fr/conseil-constitutionnel/francais/la-constitution/les-constitutions-de-la-france/constitution-de-1848-iie-republique.5106.html.

19 Sénatus-consulte du 21 mai 1870, fixant la Constitution de l'Empire // http://www. conseil-constitutionnel.fr/conseil-constitutionnel/francais/la-constitution/les-constitutionsde-la-france/constitution-de-1852-second-empire.5107.html. 
nique resta en vigueur moins de quatre mois. En septembre 1870, la IIIème République française fut proclamée. L'adoption de la Constitution républicaine prit cinq ans.

La Constitution de 1875 se composa de trois lois de nature constitutionnelle :la loi du 24 février 1875, sur l'organisation du Sénat ; la loi du 25 février 1875, sur l'organisation des pouvoirs publics ; et la loi du 16 juillet 1875, sur les rapports entre les pouvoirs publics. Les lois constitutionnelles de 1875 ne proclamèrent pas de principes d'ordre constitutionnel, ou de droits et de libertés traditionnels, de même qu'elles ne dirent rien à propos de la bannière, de l'hymne, ou des jours fériés. Les énoncés déclaratifs à caractère fondamental furent en général absents de la nouvelle Constitution. La base de la Constitution ne comporta pas du système particulier : les articles des lois constitutionnelles y furent énoncés partiellement de manière non systématique. Il n'y eut pas d'ordre préétabli de présentation des normes constitutionnelles : « une règle fut formulée lorsquion pensa à ce sujet, et des ajouts ou des conséquences, résultant de cette règle, furent inscrits plus tard, quand ils vinrent à l'esprit $»^{20}$. En conséquence, la Constitution de la IIIème République ne posséda aucune mention des droits énoncés par la Déclaration de 1789.

Pour les législateurs de 1875, le problème le plus important fut la reconnaissance d'une certaine forme de gouvernement. Dans ces conditions, la loi constitutionnelle ne comprit que ce qui fut absolument nécessaire au fonctionnement de l'administration publique. Cependant, cela ne signifia pas que l'Assemblée nationale rejeta les dispositions fondamentales de la Déclaration de 1789. À l'assemblée du Parlement du 1er février 1875, le député Henri Wallon fit la déclaration suivante : "Nous (l'Assemblée nationale) avons établi un certain nombre de dispositions constitutionnelles, sans une pensée à la formulation de déclarations philosophiques. Nos principes sont connus. Ce sont les principes de $1789 »^{21}$. Selon L. Duguitnota, "si le législateur adoptait une loi qui contrevient à l'un des principes, énoncés dans la Déclaration des droits de 1789, cette loi serait inconstitutionnelle. Nous croyons même que la Déclaration des droits de 1789 est toujours nécessaire non seulement pour le législateur ordinaire, mais aussi pour le législateur-fondateur, et que l'Assemblée nationale, formée conformément à l'article 8 de la Loi du 25 Février 1875, ne pouvait légalement émettre une loi contraire à la Déclaration de $1789 »^{22}$.

Cependant, plus tard, fin du XIXème' début du XXèmesiècle, les législateurs français adoptèrent une série de lois, contenant des dispositions sur les droits et libertés (telles que la Loi sur la liberté de réunion de 1881, la Loi sur la liberté de la presse de 1881, la Loi sur la liberté syndicale de 1901, etc...). Ces lois et un certain nombre d'autres lois sont de nos jours reconnues par le Conseil constitutionnel de la Vème République en tant que constitutionnelles. Ainsi, sur la base de la reconnaissance constitutionnelle des droits

20 Бартелеми Ж. Государственное право Франции. М.Л., 1936. С. 45.

21 Эсмен А. Общие основания конституционного права. СПб., 1898. С. 323.

22 Дюги Л. Конституционное право. Общая теория государства. С. 314. 
et libertés en France à la fin du XVIIIème et XIXème siècles, les tendances suivantes peuvent être identifiées :

Tout d'abord, l'absence presque complète de mécanismes constitutionnels pour assurer le respect des droits et des libertés.

Deuxièmement, une tendance qui peut être tracée, depuis la Constitution de l'an VIII : la réduction en déclaration des droits et des libertés (à l'exception de la Constitution de 1848).

Troisièmement, la reconnaissance constitutionnelle des droits et libertés, depuis 1789 devint un principe constitutionnel en France. Tel que formlé dans l'art. 16 de la Déclaration et selon les principes énoncés notamment par Montesquieu : "Une société où une garantie des droits n'est pas fournie et il n'y a pas de séparation des pouvoirs, n'a point de Constitution $»^{23}$.

Ainsi, le législateur français a souligné la nécessité d'une mise en œuvre effective des principes et des libertés qui y sont énoncés, ainsi que des garanties de leur réalisation dans la Constitution. Cette disposition est essentielle à la formation du constitutionnalisme français. C'est exactement selon cette interprétation que le constitutionnalisme après la révolution française est devenu une forme de légitimation des pouvoirs, à la fois républicain et monarchique.

\section{SUMMARY}

\section{Constitutional recognition of rights and freedoms in France in the late nineteenth century}

The article deals with the ideological and theoretical foundations of the French constitutionalism of the Third Republic. The author notes that the Constitution of the Third Republic was a result of the continuous struggle between the supporters of the republican form of government and monarchists in the National Assembly. A compromise, reached by legislators in 1875 , led to the uniting of different approaches to the organization of French governmental power in the Constitution. The views of Duke V. de Broglie, E. Laboulaye and L.-A. Prévost-Paradol were reflected in the Constitutional Law of the Third Republic. As a consequence, there was an acceptance of the institutions and authorities of public law specific to a dual monarchy. However, those public and legal institutions turned out to be indifferent to the form of government, that, in its turn, determined the longevity of the main constitutional and legal institutions, established in the last quarter of the nineteenth century.

Keywords: constitutionalism, the Constitution of the Third Republic, form of government, political and legal views

23 Déclaration des droits de l'homme et du Citoyen du 26 août 1789 // http://www.conseil-constitutionnel.fr/conseil-constitutionnel/francais/la-constitution/les-constitutions-de-la-france/ constitution-de-1791.5082.html 